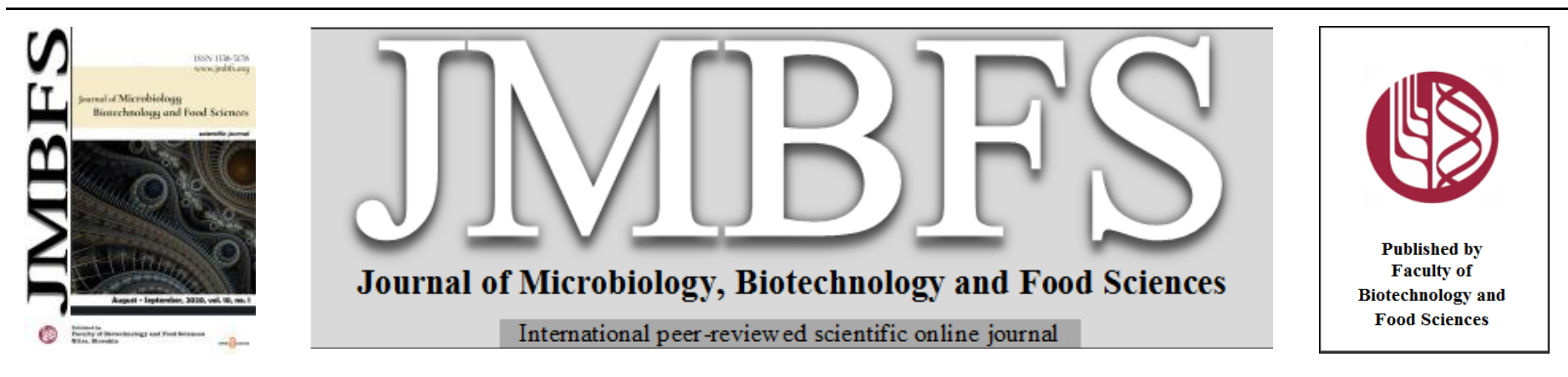

\title{
COMPARATIVE STUDY OF THE CHEMICAL COMPOSITION OF THE ESSENTIAL OIL OF ORIGANUM COMPACTUM FROM THE SEVEN REGIONS OF MOROCCO AND THEIR ANTIMICROBIAL ACTIVITY
}

\author{
Jamila Fliou $^{l}$, Ouassima Riffi ${ }^{1}$, Ali Amechrouq ${ }^{l}$, Mohammed Elhourri $^{1 *}$, Yasmine Ghouati $^{2}$ \\ Address(es): Elhourri Mohammed, \\ ${ }^{1}$ Molecular Chemistry and Natural Substances Laboratory, Moulay Ismail University, Faculty of Sciences, B.P. 11201, Zitoune, Meknes, Morocco. \\ ${ }^{2}$ Food Technology and Biochemistry Unit, Moulay Ismail University, National School of Agriculture, B.P. S/40, 50000 Meknes, Morocco.
}

*Corresponding author: med.elhourri@gmail.com

doi: 10.15414/jmbfs.2020.10.1.42-48

\section{ARTICLE INFO}

Received 5. 2. 2020

Revised 3. 3. 2020

Accepted 4. 3. 2020

Published 1. 8. 2020

Regular article

OPEN OACCESS

\section{ABSTRACT}

The aim of this work is to determine the chemical composition of the essential oil of Origanum compactum, from the region of Meknes (Morocco), and their antimicrobial effect, and on the other hand, to carry out a comparison between the chemical composition of this essential oil with the six regions of Morocco: Larache, Chefchaouen, Al Hoceïma, Tetouan, Taounate and Rabat.

The essential oils of Origanum compactum thus obtained were analyzed and identified by gas chromatography coupled with mass spectrometry (GC/MS).

To determine the similarities and dissimilarities between the chemical compositions of the essential oil of Origanum compactum from the Meknes region with the other six regions of Morocco, we performed the principal component analysis (ACP).

Regarding antimicrobial activity, the essential oils of Origanum compactum have shown significant inhibitory activity against the bacteria and molds studied.

Keywords: Origanum compactum, Chemical composition, GC/MS, ACP, Antimicrobial

\section{INTRODUCTION}

Origanum compactum is an endemic plant from Morocco and southern Spain (Jahandiez and Maire, 1932). It is common in the North and the Center of the Country. The vernacular names are «zaatar tadlawi» and «zaatar», this dialect is carried by other origins of Morocco and various thyms (Bellakhdar, 1997). It is considered by Moroccans as the real zaatar.

It is a perennial plant with a pubescent stem covered with long hairs, stem leaves oval-ovoid, hairy, inflorescences in dense and short spikes, very purple, floral bracts oval-lanceolate, rigid, leathery, large flowers, calyx with five triangular teeth and sub-equal, with ciliolated margins. This plant is found in forests, scrub and matorrals, on well-drained soils, under semi-arid and subhumid bioclimates with hot and cool variants and at the level of thermomediterranean and mesomediterranean vegetation stages (Benabid, 2000). It is distributed in the Middle Atlas, Middle Atlantic Morocco, North Atlantic Morocco and the Rif (Fennane et al., 2007).

All over Morocco, this oregano is considered a panacea. It is widely used in folk medicine due to its multiple therapeutic effects. It is mainly used as an infusion or decoction in the treatment of dysentery, colitis, gastrointestinal complaints, gastric acidity and bronchopulmonary diseases, against colds, flu, otolaryngeal diseases (O.R.L.) and bronchitis. It is also administered as a fumigation. It is used as a gargle against affections of the mouth (canker sores and gingivitis) (Bellakhdar, 1997). In the region of Zaër, the leafy stem, in infusion or decoction, is also used as a hypoglycé miant (Lahsissene et al., 2009).

In the literature, it has been reported that Origanum compactum has antibacterial (Bouhdid et al., 2008; Sfeir et al., 2013), antifungal (Salghi et al., 2013), antioxidant, anti malarial (El Babili et al., 2011), antiproliferative activities against breast cancer cells (Chaouki et al., 2010) and molluscicides (Hmamouchi et al., 2000).

\section{MATERIAL AND METHOD}

\section{Plant material}

The plant material consists of all the aerial parts of Origanum compactum growing spontaneously in the Meknes region. It was then dried in the laboratory in the dark in a well-ventilated place at room temperature. The identification of this botanical species was carried out at the biology department of the Faculty of Sciences of Meknes, Morocco.

\section{Extraction of essential oils}

The essential oil was extracted from the aerial parts of Origanum compactum by hydrodistillation using a Clevenger type device. In fact, three distillations were carried out by boiling $100 \mathrm{~g}$ of each plant material impregnated with a sufficient amount of water for three hours. The essential oil yield (volume in $\mathrm{mL}$ ) was determined relative to $100 \mathrm{~g}$ of the dry matter. Then the oil obtained was dried over anhydrous sodium sulfate $\left(\mathrm{Na}_{2} \mathrm{SO}_{4}\right)$ and stored at a temperature of $-4{ }^{\circ} \mathrm{C}$ in a dark bottle until needed.

\section{Liquid-solid chromatography on silica gel}

The essential oils obtained by hydrodistillation were fractionated by column chromatography. The operating conditions are presented below:

Column diameter: $4 \mathrm{~cm}$

Silica: 230-400 mesh (Janssen, Chimica)

Silica height: $30 \mathrm{~cm}$

Eluents: Hexane (100\%); Ether/Hexane (3/97); Ether/Hexane (5/95); Ether/Hexane (8/92); Ether/Hexane (13/87); Ether/Hexane (20/80); Ether $(100 \%)$.

\section{Chromatographic analysis}

The chromatographic analyzes were carried out on a gas chromatograph with electronic pressure regulation of the Hewlett-Packard type (HP 5890 series), equipped with a capillary column of fused silica of the VB-5 type (Methylpolysiloxane at $5 \%$ phenyl) of $30 \mathrm{~m}$ long, $0.25 \mathrm{~mm}$ in diameter and 0.25 $\mu \mathrm{m}$ film thickness, a flame ionization detector (FID) set at $260{ }^{\circ} \mathrm{C}$ and supplied with a mixture of $\mathrm{H}_{2} /$ air $^{-1}$ gas and a split-splitless injector set at $250{ }^{\circ} \mathrm{C}$. The injection mode is split (leakage ratio: 1/50, flow rate: $66 \mathrm{~mL} / \mathrm{min}^{-1}$ ). The carrier gas used is nitrogen with a flow rate of $1 \mathrm{~mL} / \mathrm{min}^{-1}$. The column temperature is programmed from 50 to $250{ }^{\circ} \mathrm{C}$ at a rate of $5{ }^{\circ} \mathrm{C} / \mathrm{min}^{-1}$. The device is controlled by a computer system of the «HP ChemStation» type managing the operation of the device and making it possible to follow the progress of the chromatographic analyzes. The fragmentation is carried out by electronic impact under a field of $70 \mathrm{ev}$. The carrier gas is helium, the flow rate of which is set at $1.4 \mathrm{~mL} / \mathrm{min}^{-1}$ These fragment ions are then separated as a function of their masse/charge ratio by the application of an electronic field (quadrupole), then collected by a 
detector. All of these fragment ions constitute the mass spectrum. The device is connected to a computer system managing a library of NIST mass spectra.

\section{Principal component analysis (PCA)}

Principal component analysis (PCA) is one of the multivariate descriptive analyzes. The purpose of using this analysis is to summarize as much information as possible while losing as little as possible to facilitate the interpretation of a large amount of initial data or to give more meaning to the reduced data.

This analysis consists in transforming the «p» initial quantitative correlated correlates into «p» quantitative variables correlated or not called «principal components».

It aims to highlight, in graphical form, the maximum of the information contained in a Data Table of a large number of descriptors, to know the amount of variance explained by the few independent main axes and to identify the relationships between variables and records. In fact, it makes it possible to obtain a representation of the point cloud in a space of reduced dimension so that the inertia carried by this space is as large as possible. It is used when it comes to describing a Table of continuous numeric variables of type «quantitative variables $\mathrm{x}$ individuals».

Statistical studies were carried out with XLSTAT Version 2014. The PCA were performed with Pearson-type matrices. The $\mathrm{CAH}$ and dendrograms were performed with dissimilarity matrices calculated in Euclidean distance and the method of aggregation chosen systematically is the average link.

\section{Biological test}

The minimum inhibitory concentrations (MIC) of essential oils were determined according to the method reported by Remmal et al. (1993) and Satrani et al (2001). Due to the immiscibility of essential oils in water and therefore in the culture medium, the emulsification was carried out using a $0.2 \%$ agar solution in order to promote the germ/compound contact. Dilutions are prepared 1/10, 1/25, $1 / 50,1 / 100,1 / 200,1 / 300$ and $1 / 500$ in the agar solution.

In test tubes each containing $13.5 \mathrm{~mL}$ of TSA (Tryptic Soy Agar) agar medium, autoclaved for 20 minutes at $121{ }^{\circ} \mathrm{C}$ and cooled to $45^{\circ} \mathrm{C}, 1.5 \mathrm{~mL}$ of each of the dilutions are added so as to obtain the final concentrations of $1 / 100$ to $1 / 5000$ $(\mathrm{v} / \mathrm{v})$. The tubes are then shaken well before being poured into petri dishes. Controls, containing only the culture medium supplemented with the $0.2 \%$ agar solution alone were prepared. The bacteria are streaked with a calibrated platinum loop to collect the same volume of inoculum. The latter is taken from a young culture in culture broth $\left(6 \mathrm{~h}\right.$ at $\left.37^{\circ} \mathrm{C}\right)$. For fungi the agar medium (MEA) is seeded with a $6 \mathrm{~mm}$ diameter fragment taken from the periphery of a funga culture in the MEA medium seven days old. Each test was repeated three times to minimize the experimental error.

\section{RESULTS AND DISCUSSION}

\section{Essential oil yield}

The aerial part of Origanum compactum provided a limpid yellow essential oil with a very strong odor with an average yield of around $3.62 \%$. The comparison of this essential oil yield with those reported in the literature, shows that Origanum compactum from the Meknes region is richer in essential oil. Indeed, this rate is higher than that found by Bakhy et al. (2014) on thirty-six samples from north-west Morocco (Rif) $(0.31 \%$ to $2.44 \%)$. This increase in yield is probably due to the altitude, the soil, the climate and the harvest period.

\section{GPC/MS analysis of the essential oil of Origanum compactum from the Meknes region}

Chromatographic analyzes of the essential oil of Origanum compactum from the Meknes region have shown the appearance of seventeen peaks relating to seventeen constituents representing approximately $88.72 \%$ of the chemical composition of the essential oil (Figure 1). This composition is characterized by the predominance of Thymol (56.41\%) and (+)-3-Carene (13.56\%). Among the different classes of essential oil are ten monoterpene hydrocarbons, a sesquiterpene hydrocarbon, four oxygenated monoterpenes and two oxygenated sesquiterpenes representing respectively $27.18 \%, 3.67 \%, 57.21 \%$, and $0.66 \%$ of the chemical composition (Table 1).

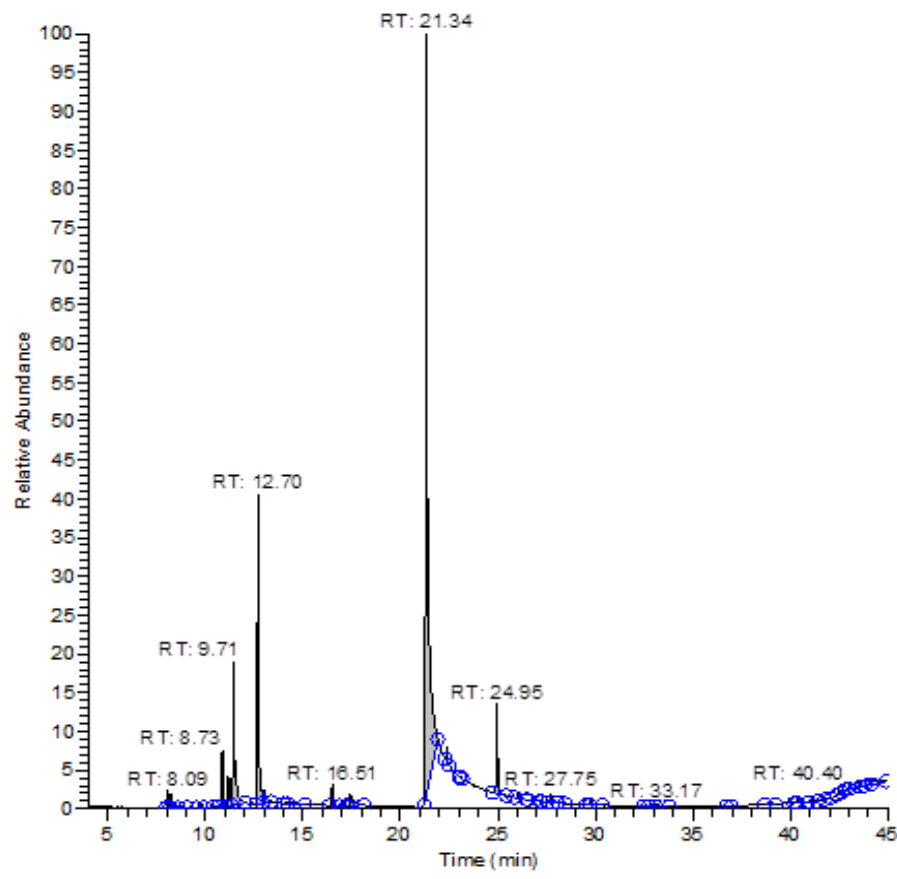

Figure 1 Chromatogram of the essential oil of Origanum compactum from the Meknes region

Table 1 Chemical composition of the essential oil of Origanum compactum from the Meknes region

\begin{tabular}{cclc}
\hline IR & Compound & Formula & Percentage (\%) \\
\hline 925 & $\alpha$-Thujene & $\mathrm{C}_{10} \mathrm{H}_{16}$ & 0.76 \\
933 & $\alpha$-Pinene & $\mathrm{C}_{10} \mathrm{H}_{16}$ & 0.61 \\
946 & Camphene & $\mathrm{C}_{10} \mathrm{H}_{16}$ & 0.11 \\
967 & Sabinene & $\mathrm{C}_{10} \mathrm{H}_{16}$ & 0.18 \\
999 & $\alpha$-Phellandrene & $\mathrm{C}_{10} \mathrm{H}_{16}$ & 8.64 \\
1011 & $(+)-3$-Carene & $\mathrm{C}_{10} \mathrm{H}_{16}$ & 13.56 \\
1051 & $\gamma$-Terpinene & $\mathrm{C}_{10} \mathrm{H}_{16}$ & 1.29 \\
1089 & $\alpha$-Terpinolene & $\mathrm{C}_{10} \mathrm{H}_{16}$ & 1.51 \\
1129 & E,Z-Alloocimene & $\mathrm{C}_{10} \mathrm{H}_{16}$ & 0.12 \\
1142 & E,E-Alloocimene & $\mathrm{C}_{10} \mathrm{H}_{16}$ & 0.40 \\
1163 & Terpinen-4-ol & $\mathrm{C}_{10} \mathrm{H}_{18} \mathrm{O}$ & 0.15 \\
1268 & Thymol & $\mathrm{C}_{10} \mathrm{H}_{14} \mathrm{O}$ & 56.41 \\
1279 & Carvacrol & $\mathrm{C}_{10} \mathrm{H}_{14} \mathrm{O}$ & 0.57 \\
1287 & p-Cymen-7-ol & $\mathrm{C}_{10} \mathrm{H}_{14} \mathrm{O}$ & 0.08 \\
1434 & $\beta$-Caryophyllene & $\mathrm{C}_{15} \mathrm{H}_{24}$ & 3.67 \\
1581 & Cedrol & $\mathrm{C}_{15} \mathrm{H}_{26} \mathrm{O}$ & 0.44 \\
1604 & Cedrenol & $\mathrm{C}_{15} \mathrm{H}_{24} \mathrm{O}$ & 0.22 \\
\hline Total & & $\mathbf{8 8 . 7 2}$ \\
\hline Monoterpene hydrocarbons & & $\mathbf{2 7 . 1 8}$ \\
Sesquiterpene hydrocarbons & & $\mathbf{3 . 6 7}$ \\
Oxygenated monoterpenes & & $\mathbf{5 7 . 2 1}$ \\
Oxygenated sesquiterpenes & & $\mathbf{0 . 6 6}$ \\
\hline TR: Retention time, $(-):$ Absence & &
\end{tabular}

In order to find new compounds of the essential oil of Origanum compactum, we chromatographed $2 \mathrm{~g}$ of the essential oil of Origanum compactum on a column in liquid phase at atmospheric pressure $(\mathrm{CPL})$. Three fractions were recovered: $\mathrm{F}_{1}$ $(0.85 \mathrm{~g}), \mathrm{F}_{2}(0.66 \mathrm{~g})$ and $\mathrm{F}_{3}(0.39 \mathrm{~g})$. Analysis by GPC/MS of the fractions $\mathrm{F}_{1}, \mathrm{~F}_{2}$ and $\mathrm{F}_{3}$ of the essential oil of Origanum compactum, made it possible to identify respectively $46.43 \%, 92.46 \%$, and $56.41 \%$ of their chemical composition.

The analysis of the different fractions allowed us to identify sixteen new compounds compared to seventeen already identified in the crude essential oil. Indeed, the fraction $F_{1}$ mainly consists of $\beta$-Caryophyllene $(19.42 \%)$ and of $\gamma$ Terpinene (11.25\%) (Figure 2), On the other hand, the fraction $\mathrm{F}_{2}$ consists mainly of Thymol $\left(91.25 \%\right.$ ) (Figure 3). For fraction $F_{3}$, the presence of $\alpha$-Terpineol $(33.66 \%)$, Cubenol (10.35\%) and Borneol (5.19\%) is noted (Figure 4). 


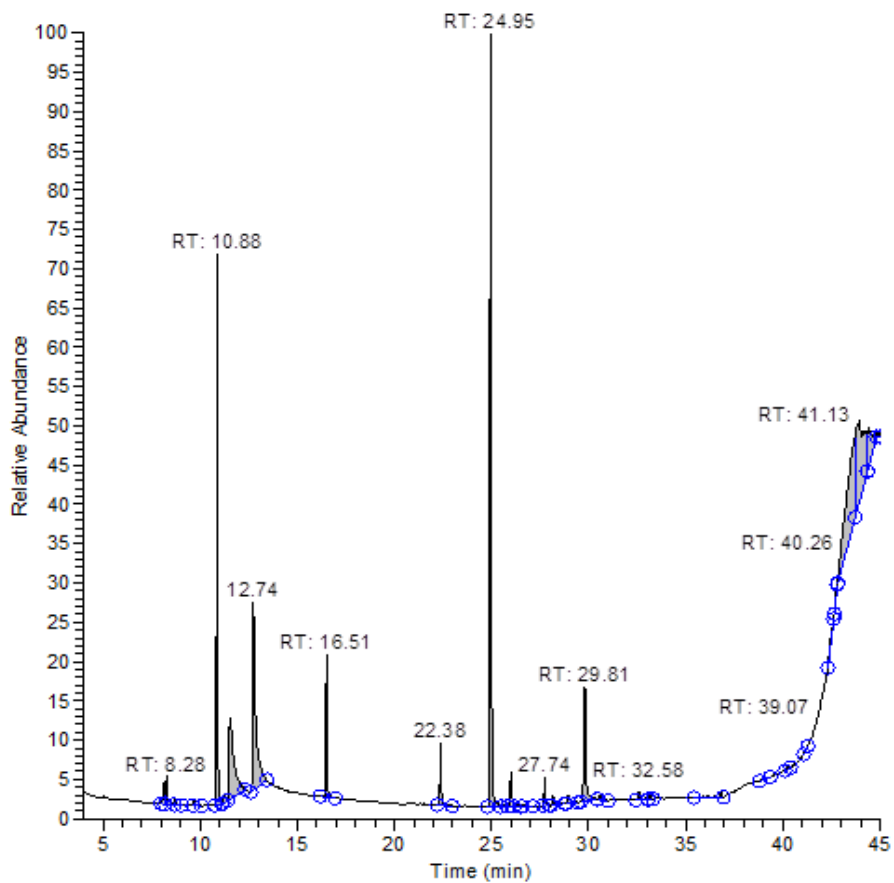

Figure 2 Chromatogram of the $\mathrm{F}_{1}$ fraction obtained by CPL of the essential oil of Origanum compactum from the Meknes region

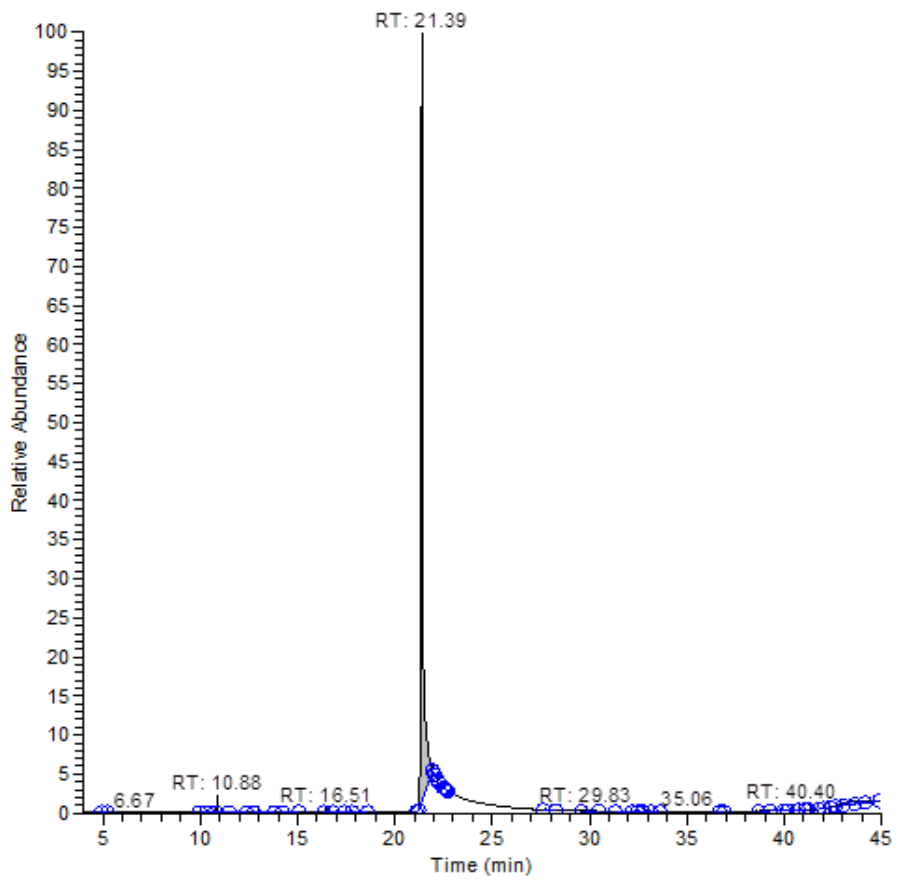

Figure 3 Chromatogram of the $\mathrm{F}_{2}$ fraction obtained by CPL of the essential oil of Origanum compactum from the Meknes region

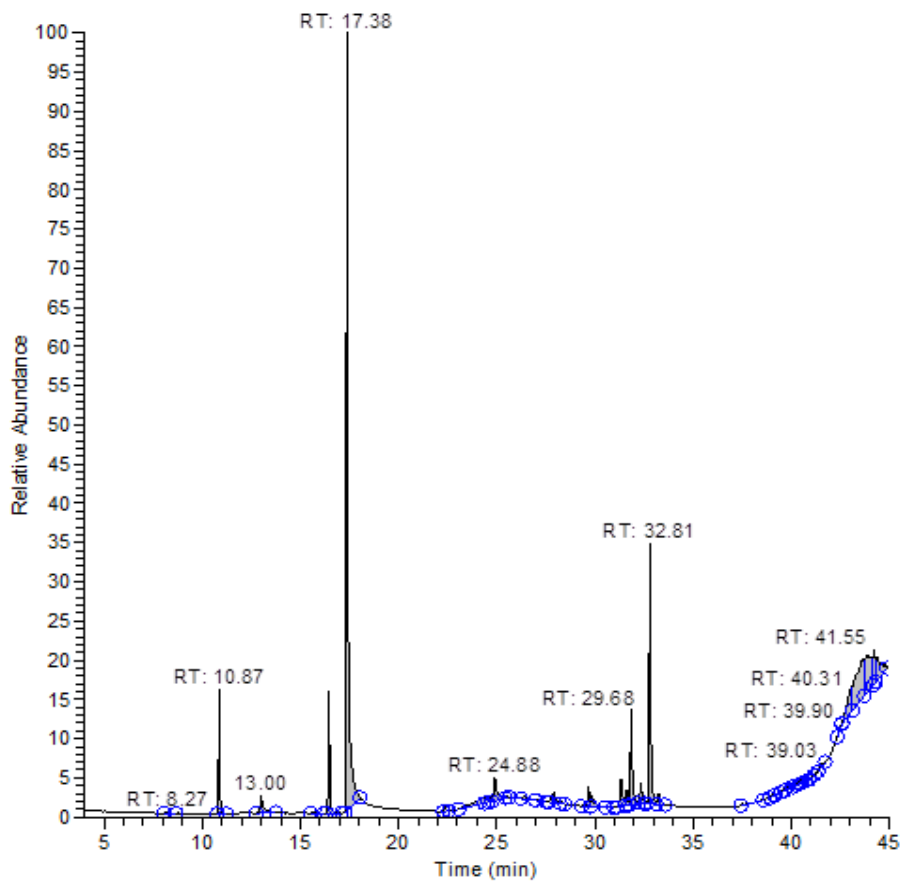

Figure 4 Chromatogram of the $\mathrm{F}_{3}$ fraction obtained by CPL of the essential oil of Origanum compactum from the Meknes region

We summarize the results obtained by gas chromatography coupled to mass spectrometry (GC/SM) of the three fractions in table 2 below:

Table 2 Chemical composition of the fractions obtained by CPL on silica gel of the essential oil of Origanum compactum from the region of Meknes

\begin{tabular}{|c|c|c|c|c|c|}
\hline \multirow{2}{*}{ IR } & \multirow{2}{*}{ Compound } & \multirow{2}{*}{ Formula } & \multicolumn{3}{|c|}{ Percentage (\%) } \\
\hline & & & $\overline{F_{1}}$ & $F_{2}$ & $\overline{F_{3}}$ \\
\hline 925 & $\alpha$-Thujene & $\mathrm{C}_{10} \mathrm{H}_{16}$ & 0.70 & - & - \\
\hline 933 & $\alpha$-Pinene & $\mathrm{C}_{10} \mathrm{H}_{16}$ & 0.97 & - & 0.14 \\
\hline 1014 & p-Cymène & $\mathrm{C}_{10} \mathrm{H}_{14}$ & 7.92 & - & - \\
\hline 1051 & $\gamma$-Terpinene & $\mathrm{C}_{10} \mathrm{H}_{16}$ & 11.25 & - & - \\
\hline 1054 & $\begin{array}{c}\text { Trans Sabinene } \\
\text { hydrate }\end{array}$ & $\mathrm{C}_{10} \mathrm{H}_{18} \mathrm{O}$ & - & - & 1.44 \\
\hline 1163 & Terpinen-4-ol & $\mathrm{C}_{10} \mathrm{H}_{18} \mathrm{O}$ & - & 0.20 & - \\
\hline 1165 & Borneol & $\mathrm{C}_{10} \mathrm{H}_{18} \mathrm{O}$ & - & - & 5.19 \\
\hline 1173 & $\alpha$-Terpineol & $\mathrm{C}_{10} \mathrm{H}_{18} \mathrm{O}$ & - & - & 33.66 \\
\hline 1242 & Carvone & $\mathrm{C}_{10} \mathrm{H}_{14} \mathrm{O}$ & - & 0.03 & - \\
\hline 1268 & Thymol & $\mathrm{C}_{10} \mathrm{H}_{14} \mathrm{O}$ & - & 91.25 & 0.43 \\
\hline 1279 & Carvacrol & $\mathrm{C}_{10} \mathrm{H}_{14} \mathrm{O}$ & - & 0.02 & 1.56 \\
\hline 1287 & p-Cymen-7-ol & $\mathrm{C}_{10} \mathrm{H}_{14} \mathrm{O}$ & - & - & 0.46 \\
\hline 1288 & p-Menth-2-en-1-ol & $\mathrm{C}_{10} \mathrm{H}_{18} \mathrm{O}$ & - & 0.28 & - \\
\hline 1420 & Longifolene & $\mathrm{C}_{15} \mathrm{H}_{24}$ & - & - & 0.90 \\
\hline 1434 & $\beta$-Caryophyllene & $\mathrm{C}_{15} \mathrm{H}_{24}$ & 19.42 & - & - \\
\hline 1436 & $\begin{array}{c}\text { trans- } \alpha- \\
\text { Bergamotene }\end{array}$ & $\mathrm{C}_{15} \mathrm{H}_{24}$ & - & - & 0.17 \\
\hline 1439 & $\alpha$-Guaiene & $\mathrm{C}_{15} \mathrm{H}_{24}$ & 1.00 & - & - \\
\hline 1524 & õ-Cadinène & $\mathrm{C}_{15} \mathrm{H}_{24}$ & 0.52 & - & - \\
\hline 1569 & $\begin{array}{l}\text { Caryophyllene } \\
\text { oxide }\end{array}$ & $\mathrm{C}_{15} \mathrm{H}_{24} \mathrm{O}$ & 4.65 & 0.43 & - \\
\hline 1576 & Spathulenol & $\mathrm{C}_{15} \mathrm{H}_{24} \mathrm{O}$ & - & - & 1.00 \\
\hline 1581 & Cedrol & $\mathrm{C}_{15} \mathrm{H}_{26} \mathrm{O}$ & - & 0.07 & 0.30 \\
\hline 1604 & Cedrenol & $\mathrm{C}_{15} \mathrm{H}_{24} \mathrm{O}$ & - & 0.18 & - \\
\hline 1642 & Cubenol & $\mathrm{C}_{15} \mathrm{H}_{26} \mathrm{O}$ & - & - & 10.35 \\
\hline 1885 & 8,14-Cedrandiol & $\mathrm{C}_{15} \mathrm{H}_{26} \mathrm{O}_{2}$ & - & - & 0.81 \\
\hline Total & & & 46.43 & 92.46 & 56.41 \\
\hline Mono & erpene hydrocarbons & & 20.84 & 0.00 & 0.14 \\
\hline Sesqu & erpene hydrocarbons & & 20.94 & 0.00 & 1.07 \\
\hline Oxyge & lated monoterpenes & & 0.00 & 91.78 & 42.74 \\
\hline Oxyge & rated sesquiterpenes & & 4.65 & 0.68 & 12.46 \\
\hline
\end{tabular}

TR: Retention time, (-): Absence, Fi: Fraction number i

By comparing the chemical composition of the essential oil of the Meknes region with that of the six other regions of Morocco (Larache, Chefchaouen, Al Hoceïma, Tetouan, Taounate and Rabat), Bakhy et al. (2014) have shown the presence of twenty-five constituents in the essential oil of Origanum compactum from the Larache region, representing approximately $95.60 \%$. The main compounds are: $\gamma$-Terpinene $(30.10 \%)$, Carvacrol $(29.70 \%)$ and p-Cymene $(11.50 \%)$. 
The identification of the various constituents revealed twelve monoterpene hydrocarbons, three sesquiterpene hydrocarbons, nine oxygenated monoterpenes and one oxygenated sesquiterpene representing respectively $48.10 \%, 1.90 \%$, $45.40 \%$ and $0.20 \%$ of the chemical composition.

Similarly Bakhy et al. (2014) have shown the appearance of twenty-five constituents representing $98.10 \%$ of the chemical composition of the essential oil of Origanum compactum from the Chefchaouen region. The main constituents are: Thymol (28.30\%), $\gamma$-Terpinene $(26.70 \%)$ and p-Cymene (11.60\%). Among these constituents are thirteen monoterpene hydrocarbons, one sesquiterpene hydrocarbon, ten oxygenated monoterpenes and one oxygenated sesquiterpene representing respectively $45.70 \%, 1.10 \%, 51.20 \%$ and $0.10 \%$ of the chemica composition of the essential oil.

Lahlou (2002) revealed that the essential oil of Origanum compactum from the Al Hoceïma region contains fourteen constituents representing approximately $97.70 \%$ of the chemical composition of the essential oil. This composition is characterized by the predominance of Carvacrol $(59.10 \%)$ and p-Cymene $(11.70 \%)$. Among the different classes of essential oil there are six monoterpene hydrocarbons, one sesquiterpene hydrocarbon, six oxygenated monoterpenes and one oxygenated sesquiterpene representing respectively $22.30 \%, 1.60 \%, 73.20 \%$ and $0.60 \%$ of the chemical composition.

Amakran et al. (2014) have shown the appearance of twenty-one constituents representing approximately $99.74 \%$ of the essential oil of Origanum compactum from the Tetouan region. The main compounds are: Carvacrol $(68.99 \%)$ and Thymol $(18.67 \%)$. The identification of the various constituents revealed nine monoterpene hydrocarbons, four sesquiterpene hydrocarbons, seven oxygenated monoterpenes and one oxygenated sesquiterpene representing respectively $7.87 \%, 1.28 \%, 90.19 \%$ and $0.40 \%$ of the chemical composition.

Ouedrhiri et al. (2016) identified twenty-two constituents representing approximately $99.45 \%$ of the essential oil of Origanum compactum from the Taounate region. The main compounds are: Carvacrol $(47.85 \%), \gamma$-Terpinene $(17.25 \%)$, Thymol $(15.75 \%)$ and p-Cymene $(8.44 \%)$. The identification of the various constituents revealed fourteen monoterpene hydrocarbons, one sesquiterpene hydrocarbon and seven oxygenated monoterpenes representing respectively $32.22 \%, 1.44 \%$ and $65.79 \%$ of the chemical composition.

El Babili et al. (2011) recorded the appearance of forty-six constituents representing $98.08 \%$ of the chemical composition of the essential oil of Origanum compactum from the region of Rabat. The main constituents are: Carvacrol (36.46\%), Thymol $(29.74 \%)$ and p-Cymene $(24.31 \%)$. Among these constituents, there are eleven monoterpene hydrocarbons, fourteen sesquiterpene hydrocarbons fourteen oxygenated monoterpenes and seven oxygenated sesquiterpenes representing respectively $27.88 \%, 1.20 \%, 67.94 \%$ and $1.06 \%$ of the chemical composition. All the results are summarized in table 3 .

Table 3 Chemical composition of the essential oils of Origanum compactum from seven regions of Morocco

\begin{tabular}{|c|c|c|c|c|c|c|c|c|c|}
\hline IR & Compound & Formula & La & Ch & Al & Te & Ta & $\mathbf{R a}$ & Me \\
\hline 925 & $\alpha$-Thujene & $\mathrm{C}_{10} \mathrm{H}_{16}$ & 1.10 & 1.20 & 0.80 & 0.09 & - & 0.03 & 0.76 \\
\hline 933 & $\alpha$-Pinene & $\mathrm{C}_{10} \mathrm{H}_{16}$ & 0.50 & 0.60 & 0.40 & 0.13 & 0.61 & 0.62 & 0.61 \\
\hline 946 & Camphene & $\mathrm{C}_{10} \mathrm{H}_{16}$ & - & 0.10 & - & - & 0.09 & 0.11 & 0.11 \\
\hline 962 & 1-Octen-3-ol & $\mathrm{C}_{8} \mathrm{H}_{16} \mathrm{O}$ & - & 0.80 & 1.10 & 0.08 & - & 0.23 & - \\
\hline 965 & 3-Octanone & $\mathrm{C}_{8} \mathrm{H}_{16} \mathrm{O}$ & 0.70 & 1.20 & - & - & 0.09 & 0.12 & - \\
\hline 967 & Sabinene & $\mathrm{C}_{10} \mathrm{H}_{16}$ & 0.10 & 0.30 & - & - & 1.06 & - & 0.18 \\
\hline 971 & $\beta$-Thujene & $\mathrm{C}_{10} \mathrm{H}_{16}$ & - & - & - & - & 0.21 & - & - \\
\hline 973 & $\beta$-Pinene & $\mathrm{C}_{10} \mathrm{H}_{16}$ & 0.10 & 0.10 & - & - & 0.21 & - & - \\
\hline 980 & 3-Octanol & $\mathrm{C}_{8} \mathrm{H}_{18} \mathrm{O}$ & - & 0.20 & - & - & - & - & - \\
\hline 983 & Myrcene & $\mathrm{C}_{10} \mathrm{H}_{16}$ & 1.70 & 1.90 & 1.20 & 0.44 & 1.42 & 0.33 & - \\
\hline 999 & $\alpha$-Phellandrene & $\mathrm{C}_{10} \mathrm{H}_{16}$ & 0.20 & 0.20 & - & 0.05 & 0.24 & 0.04 & 8.64 \\
\hline 1001 & 2-Carene & $\mathrm{C}_{10} \mathrm{H}_{16}$ & - & - & - & - & 0.12 & - & - \\
\hline 1010 & $\alpha$-Terpinene & $\mathrm{C}_{10} \mathrm{H}_{16}$ & 2.20 & 2.30 & 1.10 & 0.48 & 2.19 & 0.36 & - \\
\hline 1011 & (+)-3-Carene & $\mathrm{C}_{10} \mathrm{H}_{16}$ & - & - & - & - & 0.07 & 0.04 & 13.56 \\
\hline 1014 & p-Cymene & $\mathrm{C}_{10} \mathrm{H}_{14}$ & 11.50 & 11.60 & 11.70 & 2.53 & 8.44 & 24.31 & - \\
\hline 1023 & Limonene & $\mathrm{C}_{10} \mathrm{H}_{16}$ & 0.30 & 0.40 & - & 0.11 & 0.25 & - & - \\
\hline 1023 & $\beta$-Phellandrene & $\mathrm{C}_{10} \mathrm{H}_{16}$ & 0.20 & 0.20 & - & 0.06 & - & - & - \\
\hline 1038 & (E)- $\beta$-Ocimene & $\mathrm{C}_{10} \mathrm{H}_{16}$ & 0.10 & 0.10 & - & - & 0.06 & - & - \\
\hline 1051 & $\gamma$-Terpinene & $\mathrm{C}_{10} \mathrm{H}_{16}$ & 30.10 & 26.70 & 7.10 & 3.98 & 17.25 & 1.10 & 1.29 \\
\hline 1054 & trans Sabinene hydrate & $\mathrm{C}_{10} \mathrm{H}_{18} \mathrm{O}$ & 0.30 & 0.30 & - & - & - & - & - \\
\hline 1063 & 3-Nonanone & $\mathrm{C}_{9} \mathrm{H}_{18} \mathrm{O}$ & 0.10 & - & - & - & - & - & - \\
\hline 1084 & Linalool & $\mathrm{C}_{10} \mathrm{H}_{18} \mathrm{O}$ & 1.00 & 1.70 & 2.00 & 1.10 & 1.36 & 0.57 & - \\
\hline 1086 & trans- Linalool oxide & $\mathrm{C}_{10} \mathrm{H}_{18} \mathrm{O}_{2}$ & - & - & - & - & - & 0.01 & - \\
\hline 1088 & Dimethyl styrene & $\mathrm{C}_{10} \mathrm{H}_{12}$ & - & - & - & - & - & 0.13 & - \\
\hline 1089 & $\alpha$-Terpinolene & $\mathrm{C}_{10} \mathrm{H}_{16}$ & - & - & - & - & - & - & 1.51 \\
\hline 1108 & 1,3,8-p-Menthatriene & $\mathrm{C}_{10} \mathrm{H}_{14}$ & - & - & - & - & - & 0.81 & - \\
\hline 1129 & E,Z-Alloocimene & $\mathrm{C}_{10} \mathrm{H}_{16}$ & - & - & - & - & - & - & 0.12 \\
\hline 1142 & E,E-Alloocimene & $\mathrm{C}_{10} \mathrm{H} 16$ & - & - & - & - & - & - & 0.40 \\
\hline 1143 & Camphre & $\mathrm{C}_{10} \mathrm{H}_{16} \mathrm{O}$ & - & - & - & - & - & - & - \\
\hline 1163 & Terpinen-4-ol & $\mathrm{C}_{10} \mathrm{H}_{18} \mathrm{O}$ & 0.20 & 0.30 & 0.90 & 0.58 & - & - & 0.15 \\
\hline 1165 & Borneol & $\mathrm{C}_{10} \mathrm{H}_{18} \mathrm{O}$ & - & - & - & 0.19 & 0.15 & 0.03 & - \\
\hline 1173 & $\alpha$-Terpineol & $\mathrm{C}_{10} \mathrm{H}_{18} \mathrm{O}$ & 4.10 & 6.80 & 1.00 & 0.58 & - & & - \\
\hline 1183 & p-Cymen-8-ol & $\mathrm{C}_{10} \mathrm{H}_{14} \mathrm{O}$ & - & - & - & - & 0.43 & 0.14 & - \\
\hline 1194 & Myrtenol & $\mathrm{C}_{10} \mathrm{H}_{16} \mathrm{O}$ & - & - & - & - & 0.16 & - & - \\
\hline 1226 & Carvacryl methyl oxide & $\mathrm{C}_{11} \mathrm{H}_{16} \mathrm{O}$ & 7.10 & 4.90 & - & - & - & - & - \\
\hline 1242 & Carvone & $\mathrm{C}_{10} \mathrm{H}_{14} \mathrm{O}$ & - & - & - & - & - & - & - \\
\hline 1244 & Methyl carvacrol & $\mathrm{C}_{11} \mathrm{H}_{16} \mathrm{O}$ & - & - & - & - & - & 0.06 & - \\
\hline 1252 & Piperitone & $\mathrm{C}_{10} \mathrm{H}_{16} \mathrm{O}$ & - & - & - & - & - & 0.03 & - \\
\hline 1263 & Geranial & $\mathrm{C}_{10} \mathrm{H}_{16} \mathrm{O}$ & - & - & - & - & - & 0.04 & - \\
\hline 1268 & Thymol & $\mathrm{C}_{10} \mathrm{H}_{14} \mathrm{O}$ & 2.20 & 28.30 & 9.10 & 18.67 & 15.75 & 29.74 & 56.41 \\
\hline 1279 & Carvacrol & $\mathrm{C}_{10} \mathrm{H}_{14} \mathrm{O}$ & 29.70 & 6.70 & 59.10 & 68.99 & 47.85 & 36.46 & 0.57 \\
\hline 1287 & p-Cymen-7-ol & $\mathrm{C}_{10} \mathrm{H}_{14} \mathrm{O}$ & - & - & - & - & - & & 0.08 \\
\hline 1288 & p-menth-2-en-1-ol & $\mathrm{C}_{10} \mathrm{H}_{18} \mathrm{O}$ & & & & & & & \\
\hline 1364 & Piperitenone & $\mathrm{C}_{10} \mathrm{H}_{14} \mathrm{O}$ & - & - & - & - & - & 0.44 & - \\
\hline 1367 & Eugenol & $\mathrm{C}_{10} \mathrm{H}_{12} \mathrm{O}_{2}$ & - & - & - & - & - & 0.06 & - \\
\hline 1387 & $\alpha$-Copaene & $\mathrm{C}_{15} \mathrm{H}_{24}$ & - & - & - & - & - & 0.02 & - \\
\hline 1420 & Longifolene & $\mathrm{C}_{15} \mathrm{H}_{24}$ & & & & & & & \\
\hline 1418 & Caryophyllene & $\mathrm{C}_{15} \mathrm{H}_{24}$ & 1.70 & 1.10 & 1.60 & 1.09 & 1.44 & - & - \\
\hline 1421 & $\alpha$-Gurjunene & $\mathrm{C}_{15} \mathrm{H}_{24}$ & - & - & - & - & - & 0.02 & - \\
\hline 1434 & $\beta$-Caryophyllene & $\mathrm{C}_{15} \mathrm{H}_{24}$ & - & - & - & - & - & 0.78 & 3.67 \\
\hline 1436 & trans- $\alpha$-Bergamotene & $\mathrm{C}_{15} \mathrm{H}_{24}$ & - & - & - & 0.06 & - & - & - \\
\hline 1439 & $\alpha$-Guaiene & $\mathrm{C}_{15} \mathrm{H}_{24}$ & & & & & & & \\
\hline 1451 & $\alpha$-Humulene & $\mathrm{C}_{15} \mathrm{H}_{24}$ & 0.10 & - & - & 0.06 & - & 0.05 & - \\
\hline 1470 & allo-Aromodendrene & $\mathrm{C}_{15} \mathrm{H}_{24}$ & - & - & - & - & - & 0.02 & - \\
\hline
\end{tabular}




\begin{tabular}{|c|c|c|c|c|c|c|c|c|c|}
\hline 1486 & $\gamma$-Muurolene & $\mathrm{C}_{15} \mathrm{H}_{24}$ & - & - & - & - & - & 0.02 & - \\
\hline 1494 & $\beta$-Ionone & $\mathrm{C}_{13} \mathrm{H}_{20} \mathrm{O}$ & - & - & - & - & - & 0.01 & - \\
\hline 1500 & $\beta$-Bisabolene & $\mathrm{C}_{15} \mathrm{H}_{24}$ & 0.10 & - & - & - & - & 0.02 & - \\
\hline 1506 & $\alpha$-Muurolene & $\mathrm{C}_{15} \mathrm{H}_{24}$ & - & - & - & - & - & 0.02 & - \\
\hline 1509 & Ledene & $\mathrm{C}_{15} \mathrm{H}_{24}$ & - & - & - & - & - & 0.02 & - \\
\hline 1520 & $\gamma$-Cadinene & $\mathrm{C}_{15} \mathrm{H}_{24}$ & - & - & - & - & - & 0.07 & - \\
\hline 1524 & õ-Cadinene & $\mathrm{C}_{15} \mathrm{H}_{24}$ & - & - & - & 0.07 & - & 0.10 & - \\
\hline 1541 & $\alpha$-Cadinene & $\mathrm{C}_{15} \mathrm{H}_{24}$ & - & - & - & - & - & 0.01 & - \\
\hline 1551 & $\alpha$-Calacorene & $\mathrm{C}_{15} \mathrm{H}_{20}$ & - & - & - & - & - & 0.01 & - \\
\hline 1569 & Caryophyllene oxide & $\mathrm{C}_{15} \mathrm{H}_{24} \mathrm{O}$ & 0.20 & 0.10 & - & 0.40 & - & 0.86 & - \\
\hline 1576 & Spathulenol & $\mathrm{C}_{15} \mathrm{H}_{24} \mathrm{O}$ & - & - & 0.60 & - & - & 0.03 & - \\
\hline 1581 & Cedrol & $\mathrm{C}_{15} \mathrm{H}_{26} \mathrm{O}$ & - & - & - & - & - & & 0.44 \\
\hline 1604 & Cedrenol & $\mathrm{C}_{15} \mathrm{H}_{24} \mathrm{O}$ & - & - & - & - & - & & 0.22 \\
\hline 1611 & Humulene epoxide & $\mathrm{C}_{15} \mathrm{H}_{24} \mathrm{O}$ & - & - & - & - & - & 0.05 & - \\
\hline 1641 & Caryophylla 4(14), 8(15) dien-5-ol & $\mathrm{C}_{15} \mathrm{H}_{26} \mathrm{O}$ & - & - & - & - & - & 0.03 & - \\
\hline 1642 & Cubenol & $\mathrm{C}_{15} \mathrm{H}_{26} \mathrm{O}$ & - & - & - & - & - & - & - \\
\hline 1647 & T-Cadinol & $\mathrm{C}_{15} \mathrm{H}_{26} \mathrm{O}$ & - & - & - & - & - & 0.02 & - \\
\hline 1653 & $\alpha$-Cadinol & $\mathrm{C}_{15} \mathrm{H}_{26} \mathrm{O}$ & - & - & - & - & - & 0.01 & - \\
\hline 1668 & 14-hydroxy-9-epi-(E)-caryophyllene & $\mathrm{C}_{15} \mathrm{H}_{24} \mathrm{O}$ & - & - & - & - & - & 0.06 & - \\
\hline 1680 & Cadalene & $\mathrm{C}_{15} \mathrm{H}_{18}$ & - & - & - & - & - & 0.04 & - \\
\hline 1683 & Bisabolol & $\mathrm{C}_{15} \mathrm{H}_{26} \mathrm{O}$ & - & - & - & - & - & - & - \\
\hline 1885 & 8,14-cedrandiol & $\mathrm{C}_{15} \mathrm{H}_{26} \mathrm{O}_{2}$ & - & - & - & - & - & - & - \\
\hline \multicolumn{3}{|l|}{ Total } & 95.60 & 98.10 & 97.70 & 99.74 & 99.45 & 98.08 & 88.72 \\
\hline \multicolumn{3}{|c|}{ Monoterpene hydrocarbons } & 48.10 & 45.70 & 22.30 & 7.87 & 32.22 & 27.88 & 27.18 \\
\hline \multicolumn{3}{|c|}{ Sesquiterpene hydrocarbons } & 1.90 & 1.10 & 1.60 & 1.28 & 1.44 & 1.20 & 3.67 \\
\hline \multicolumn{3}{|c|}{ Oxygenated monoterpenes } & 45.40 & 51.20 & 73.20 & 90.19 & 65.79 & 67.93 & $\mathbf{5 7 . 2 1}$ \\
\hline \multicolumn{3}{|c|}{ Oxygenated sesquiterpenes } & 0.20 & 0.10 & 0.60 & 0.40 & - & 1.06 & 0.66 \\
\hline \multicolumn{3}{|c|}{ Ester } & - & - & - & - & - & 0.01 & - \\
\hline
\end{tabular}

La: Larache, Ch: Chefchaouen, Al: Al Hoceïma, Te: Tetouan, Ta: Taounate, Ra: Rabat, Me: Meknes, TR: Retention time, (-): Absence

Principal component analysis (PCA) for the main compound of Origanum compactum essential oil from seven regions of Morocco

The analysis of the links between the chemical composition of the essential oils of Origanum compactum from the Meknes region and six other regions of Morocco: Larache, Chefchaouen, Al Hoceïma, Tétouan, Taounate and Rabat was carried out using this method (PCA), only the discriminating variables were taken into account.

To carry out this analysis, two first factorial axes were chosen. The dispersion of the Origanum compactum species in the plane formed by these two axes in relation to the chosen variables explains $72.73 \%$ of the variability, including $47.94 \%$ on the first axis and $24.79 \%$ on the second axis (Figure 5).

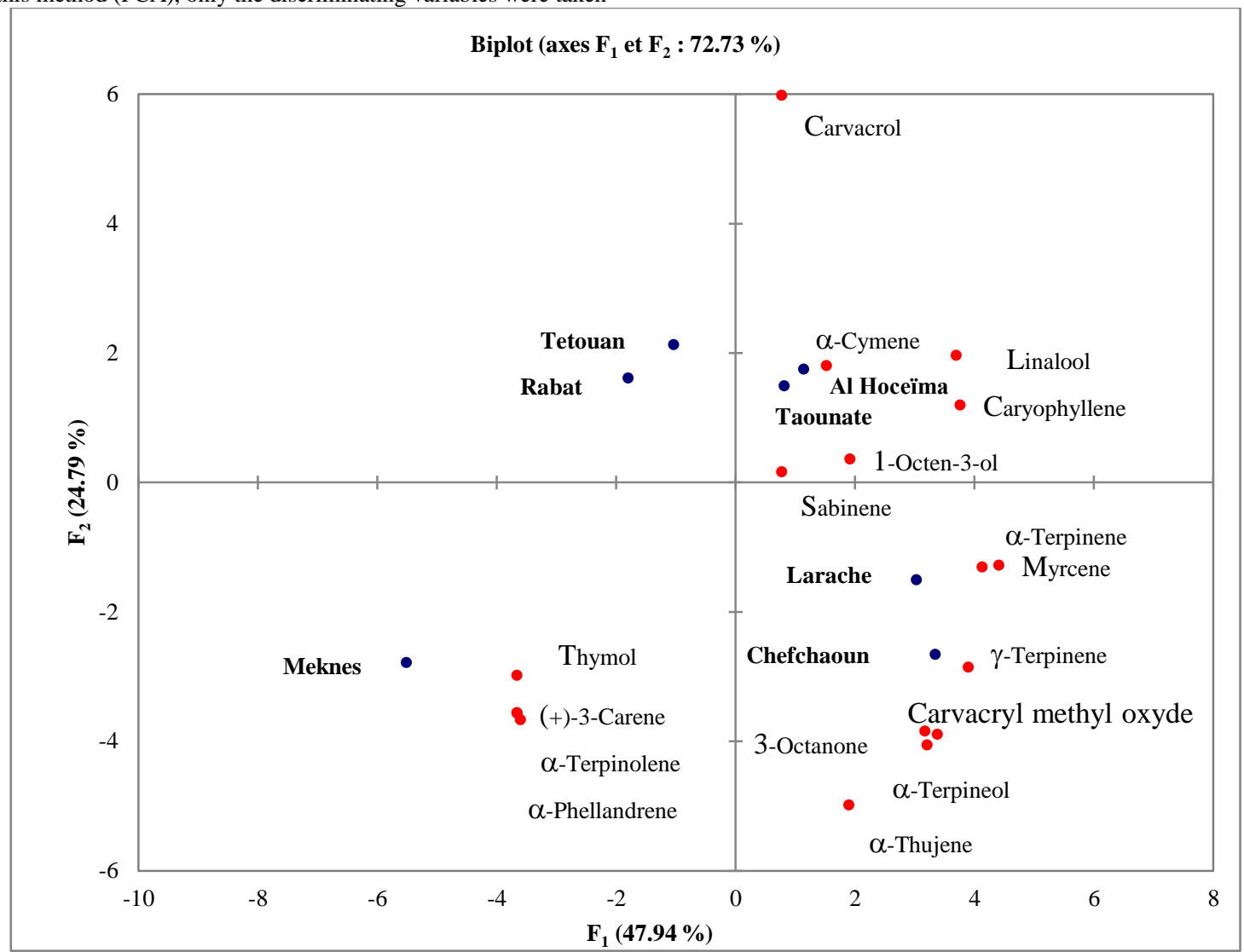

Figure 5 Principal component analysis for the main essential oil compound of Origanum compactum from seven regions of Morocco

This figure shows the separation of four groups in the two axis systems

- The group formed by Origanum compactum from the Meknes region is characterized by the high rate of Thymol and (+)-3-Carene,
- Origanum compactum from Larache and Chefchaouen forms a group close to Origanum compactum from Meknes with a high Thymol content. The high level of $\gamma$-Terpinene made it possible to separate this group from the other groups,

- The group formed by Origanum compactum from Tetouan and Rabat is close to Meknes by a high rate in Thymol and close to the group formed by Origanum 
compactum from the regions of Al Hoceïma and Taounate by a high rate in Carvacrol,

- The group formed by Origanum compactum from Al Hoceïma and Taounate is characterized by high levels of $\alpha$-Cymene and Carvacrol. This group is close to the group formed by Origanum compactum from the regions of Tetouan and Rabat by a high content of Carvacrol.

The dendrograms have clearly visualized the links between Origanum compactum of the seven regions studied (Figure 6).

\section{Antimicrobial activity of Origanum compactum}

The essential oil of Origanum compactum has a significant inhibitory activity against the bacteria and molds studied. The result of this antimicrobial and antifungal activity is presented in table 4 .

The results of the Table show that the essential oil of Origanum compactum inhibited the growth of $B$. cereus and $S$. aureus from a low concentration of the order of $0.30 \mathrm{mg} / \mathrm{mL}$, while E. coli, E. amylovora, P. savastoni and S. thyphi are inhibited from a concentration of $0.45 \mathrm{mg} / \mathrm{mL}$.

The seven molds showed different sensitivity behavior towards the essential oil of Origanum compactum. P. expansum is the most sensitive, with an MIC of 0.30 $\mathrm{mg} / \mathrm{mL}$, while A. alternata, B. cinerea, P. digitatum, P. italicum, V. dahlea and A. niger were inhibited from a concentration of $0.45 \mathrm{mg} / \mathrm{mL}$.

The transfer of the mold discs to fresh MA medium has shown that the essential oil has a fungicidal effect on the mycelial growth of all molds from a concentration of $3.64 \mathrm{mg} / \mathrm{mL}$, while an effect is obtained. Fungistatic from the essential oil concentration of $0.45 \mathrm{mg} / \mathrm{mL}$.

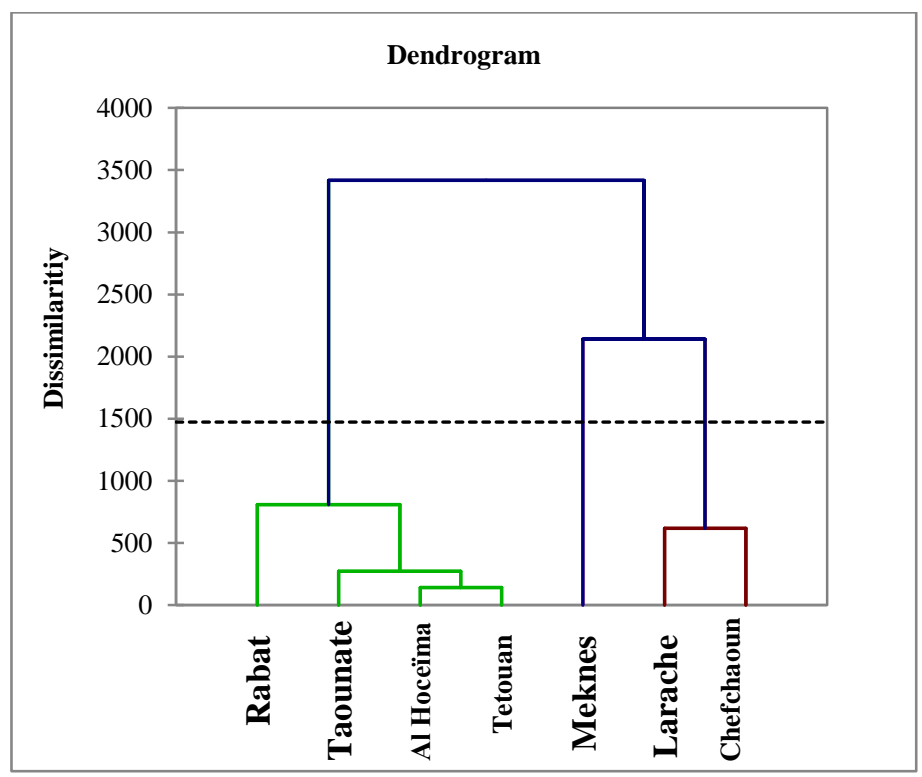

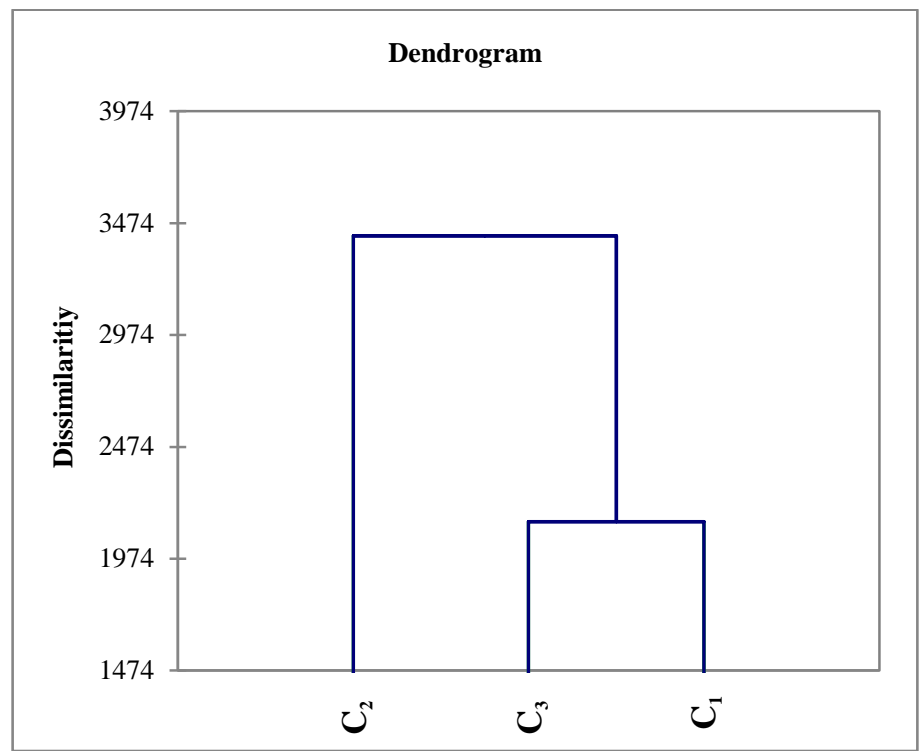

Figure 6 Dendrograms obtained from the analysis of the chemical composition of the essential oils of Origanum compactum from the seven regions of Morocco

The antifungal activity of essential oil from Origanum compactum has been demonstrated by several studies; against: the species of Aspergillus and A. niger (Charai et al., 1996), P. italicum, A. alternata (Sakkas and Papadopoulou, 2017), against Gram + bacteria and Gram- bacteria and also against resistant strains of E. coli (Ben Hammou et al., 2011; Bouhdid et al., 2008).

Laghmouchi et al., (2018) reported that the MIC for S. aureus decreases with increasing concentration of the essential of Origanum compactum. So this oil can be used as a substitute for chemical preservatives for the control of $S$. aureus: agent responsible for poisoning through the consumption of contaminated meat products. Antimicrobial activity has also been confirmed by other work (Bouyahya et al., 2016; Nayely et al., 2017; Lu et al., 2018; Teodora and Ralitsa, 2016)

Table 4 Minimum inhibitory concentrations of Origanum compactum essential oil on thirteen microorganisms

\begin{tabular}{lcccccccc}
\hline Concentration & $\mathbf{1 / 1 0 0}$ & $\mathbf{1 / 2 5 0}$ & $\mathbf{1 / 5 0 0}$ & $\mathbf{1 / 1 0 0 0}$ & $\mathbf{1 / 2 0 0 0}$ & $\mathbf{1 / 3 0 0 0}$ & $\mathbf{1 / 5 0 0 0}$ & Witness \\
\hline (mg/mL) & $\mathbf{9 . 1 0}$ & $\mathbf{3 . 6 4}$ & $\mathbf{1 . 8 2}$ & $\mathbf{0 . 9 1}$ & $\mathbf{0 . 4 5}$ & $\mathbf{0 . 3 0}$ & $\mathbf{0 . 1 8}$ & $\mathbf{0 . 0 0}$ \\
\hline Bacteria & & & & & & & & \\
\hline B. cereus & - & - & - & - & - & - & + & + \\
E. coli & - & - & - & - & - & + & + & + \\
E. amylovora & - & - & - & - & - & + & + & + \\
P.savastanoi & - & - & - & - & - & + & + & + \\
S.aureus & - & - & - & - & - & - & + & + \\
S. thyphi & - & - & - & - & - & + & + & + \\
\hline Mushrooms & & & - & & & & & + \\
\hline A. alternata & - & - & - & - & - & + & + & + \\
A. niger & - & - & - & - & - & + & + & + \\
B. cinerea & - & - & - & - & - & + & + & + \\
P. digitatum & - & - & - & - & - & + & + & + \\
P. expansum & - & - & - & - & - & - & + & + \\
P. italicum & - & - & - & - & - & + & + & + \\
V.dahlea & - & - & - & - & - & + & + & + \\
\hline
\end{tabular}

(-): Inhibition, (+): Growth

\section{CONCLUSION}

The results showed that the essential oils of the Larache region are of the $\gamma$ Terpinene chemotype with a percentage of $30.10 \%$ and of the Chafchaoun and Meknes regions with the percentages $28.30 \%$ and $56.41 \%$. Regions of Al Hoceïma, Tétouan, Taounate and Rabat have the Carvacrol chemotype with the percentages $59.10 \%, 68.99 \%, 47.85 \%$ and $36.46 \%$ respectively.

In terms of antimicrobial activity, essential oils extracted from Origanum compactum have shown significant inhibition of the six bacteria and seven molds studied from a concentration of $0.45 \mathrm{mg} / \mathrm{mL}$

At the end of this study, the essential oils of Origanum compactum can be recommended as antimicrobials against certain pathogenic microorganisms and 
as alternatives to synthetic antibiotics. This approach can help reduce the amount of synthetic antibiotics applied, and subsequently decrease the negative impact of synthetic agents, such as residues, resistance and environmental pollution.

\section{REFERENCES}

Jahandiez, E. \& Maire, R. (1931, 1932, 1934). Catalogue des Plantes du Maroc. 3 Tomes, Editions Lechevalier, Paris, 913. https://www.scirp.org/(S(351jmbntvnsjt1aadkposzje))/reference/ReferencesPaper S.aspx?ReferenceID $=1216567$

Bellakhdar, J. (1997). La pharmacopée marocaine traditionnelle, médecine arabe ancienne et savoirs populaires. In: Ibis Press (ed), Berwick Maine, Paris, p. 764 https://www.persee.fr/doc/horma_0984-

2616_1998_num_35_1_1707_t1_0319_0000_1

Benabid, A. (2000). Flore et écosystèmes du Maroc. Evaluation et préservation de la biodiversité. Ibis Press, Paris https://www.amazon.fr/\%C3\%A9cosyst\%C3\%A8mes-Maroc-Evaluation-

pr\%C3\%A9servation-biodiversit\%C3\%A9/dp/2910728137

Fennane, M., Ibn Tattou, M., Ouyahya, A., \& El Oualidi, J. (2007). (Ed.), Flore pratique du Maroc. Vol II, Trav. Inst. Sci., sér. Bot. 38, Rabat. http://www.herbmedit.org/flora/9-113.pdf

Lahsissene, H., Kahouadji, A., Tijane, M., \& Hseini, S. (2009). Catalogue des plantes médicinales utilisées dans la région de zaër (Maroc occidental). Lejeunia, BE ISSN 0457-4184. https://popups.uliege.be/0457 4184/index.php?id=713\&file $=1 \&$ pid $=701$

Bouhdid, S., Skali, S.N., Idaomar, M., Zhiri, A., Baudoux, D., Amensour, M., \& Abrini, J. (2008). Antibacterial and antioxidant activities of Origanum compactum essential oil. African Journal of Biotechnology, 7, 1563-1570. file:///C:/Users/MedSim/Downloads/58723-104998-1-PB.pdf

Sfeir, J., Lefrançois, C., Baudoux, D., Derbré, S., \& Licznar, P. (2013). In Vitro Antibacterial Activity of Essential Oils againstStreptococcus pyogenes. Evidence-based Complementary and Alternative Medicine, 1-9. https://dx.doi.org/10.1155/2013/269161

Fadel, F., Ben Hmamou, D., Salghi, R., Chebli, B., Benali, O., Zarrouk, A. Ebenso, E.E., Chakir, A., \& Hammouti, B. (2013). Antifungal Activity and AntiCorrosion Inhibition of Origanum Compactum Extracts. International Journal of Electrochemical Science, $\quad 8, \quad 11019-11032$. http://www.electrochemsci.org/papers/vol8/80911019.pdf

El Babili, F., Bouajila, J., Souchard, J.P., Bertrand, C., Bellvert, F., Fouraste, I., Moulis, C., \& Valentin, A. (2011). Oregano: chemical analysis and evaluation of its antimalarial, antioxidant, and cytotoxic activities. Journal of Food Science, 76, 512-518. https://dx.doi.org/10.1111/j.1750-3841.2011.02109.x

Chaouki, W., Leger, D.Y., Eljastimi, J., Beneytout, J.L., \& Hmamouchi, M (2010). Antiproliferative effect of extracts from Aristolochia baetica and Origanum compactum on human breast cancer cell line MCF-7. Pharmaceutical Biology, 48, 269-274. https://dx.doi.org/10.3109/13880200903096588

Hmamouchi, M., Lahlou, M., \& Agoumi, A. (2000). Molluscicidal activity of some Moroccan medicinal plants. Fitoterapia, 71, 308-314 https://doi.org/10.1016/S0367-326X(99)00152-5

Remmal, A., Bouchikhi, T., Rhayour, K., Ettayebi, M., \& Elaraki, A.T. (1993) Impoved method for determination of antimicrobial activity of essential oils in agar medium. Journal of Essential Oil Research, 5, 179-184. https://doi.org/10.1080/10412905.1993.9698197

Satrani, B., Farah, A., Fechtal, M., Talbi, M., Blaghen, M., \& Chaouch, A (2001). Composition chimique et activité antimicrobienne des huiles essentielles de Satureja calamintha et Satureja alpina du Maroc. Annales des falsifications et de l'expertise chimique et toxicologique, 94, 241-250. https://popups.uliege.be/1780-4507/index.php?id=17094\&file=1\&pid=5218

Bakhy, K., Benlhabib, O., Bighelli, A., Casanova, J., Tomi, F., \& Al Faiz, C (2014). Yield and chemical variability of the essential oil isolated from aerial parts of wild Origanum compactum Benth. From Moroccan Western Rif American Journal of Essential Oils and Natural Products, 1, 9-17. http://www.essencejournal.com/pdf/2014/vol1issue4/PartA/1-268.pdf

Lahlou, M. (2002). Potential of Origanum Compactum as a cercaricide in Morocco. Annals of Tropical Medicine \& Parasitology, 96, 587-593. https://doi.org/10.1179/000349802125001447

Amakran, A., Hamoudane, M., Ramdan, B., Ben mehdi, N., Ben mrid, R., Lesicki, M., Figueredo, G., Lamarti, A., \& Nhiri, M. (2014). Study of antioxidant, antiglyquant and phytochemical properties of Origanum compactum essential oil from tetouan. Sciencelib editions mersenne, 6, $\mathrm{n}^{\circ} 140102$ ISSN 2111-4706.

Ouedrhiri, W., Balouiri, M., Bouhdid, S., Moja, S., Ouazzani, F., Taleb, C.M., \& Greche, H. (2016). Mixture design of Origanum compactum, Origanum majorana and Thymus serpyllum essential oils: Optimization of their antibacterial effect. $\begin{array}{lllll}\text { Industrial Crops } \quad \text { and } & \text { Products, } & \text { 89, }\end{array}$ https://doi.org/10.1016/j.indcrop.2016.04.049

Charai, M., Mosaddak, M., \& Faid, M. (1996). Chemical Composition and Antimicrobial Activities of Two Aromatic Plants: Origanum majorana L. and O. compactum Benth. Journal of Essential Oil Research, 8, 657-664. https://doi.org/10.1080/10412905.1996.9701036
Sakkas, H., \& Papadopoulou, C. (2017). Antimicrobial Activity of Basil, Oregano, and Thyme Essential Oils. Journal of Microbiology and Biotechnology, 27, 429-438. https://doi.org/10.4014/imb.1608.08024

Ben Hammou, F., Skali, S.N., Idaomar, M., \& Abrini, J. (2011). The antimicrobial effect of Origanum compactum essential oil, nisin and their combination against Escherichia coli in tryptic soy broth (TSB) and in sheep natural sausage casings during storage at 25 and 7 C. African Journal of Biotechnology, 10, 71. https://doi.org/10.5897/AJB11.1046

Laghmouchi, Y., Belmehdi, O., Senhaji, N.S., \& Abrini, J. (2018). Chemical composition and antibacterial activity of Origanum compactum Benth. essential oils from different areas at northern Morocco. South African Journal of Botany 115, 120-125. https://doi.org/10.1016/j.sajb.2018.02.002

Bouyahya, A., Abrini, J., Edaoudi, F., Et-Touys, A., Bakri, Y., \& Dakka, N. (2016). Origanum compactum Benth: A Review on Phytochemistry and Pharmacological Properties. Medicinal \& Aromatic Plants, 5(4), 1-6. https://doi.org/10.4172/2167-0412.1000252

Nayely, L.L., Erick, P.G.G., Gabriela, V.O., \& Heredia, J.B. (2017). Essential Oils of Oregano: Biological Activity beyond Their Antimicrobial Properties. Molecules, 22, 989. https://doi.org/10.3390/molecules22060989

Lu, M., Clinton, K.M., \& Mei, X.Wu. (2018). Bactericidal Property of Oregano Oil Against Multidrug-Resistant Clinical Isolates. Original research, 1-14 https://doi.org/10.3389/fmicb.2018.02329

Teodora, P.P., \& Ralitsa, B. (2016). Antimicrobial Activity in vitro of Essentia Oils from Oregano (Origanum compactum L.) and Thyme (Thymus vulgaris L.) International Journal of Current Microbiology and Applied Sciences, 5, 57-68. http://dx.doi.org/10.20546/ijcmas.2016.510.008 